\title{
Clinical Predictors of Laryngeal Preservation Rate in Stage III-IV Laryngeal Cancer and Hypopharyngeal Cancer Patients Treated with Organ Preservation
}

\author{
Kanograt Tangsriwong ${ }^{1 *}$, Tastsanachart Jitreetat ${ }^{2}$
}

\begin{abstract}
Purpose: To determine factors affecting laryngeal preservation rate in laryngeal and hypopharyngeal cancer patients treated with organ preservation. Material and Methods: Retrospective study examining stage III to IV laryngeal and hypopharyngeal cancer patients who have been treated with organ preservation. Conventional radiation must be applied in all patients with minimum dose of 45 Gray. Weekly or triweekly chemotherapy can be adding during radiation. Salvage surgery should be considered in residual disease or local recurrence. Kaplan-Meier was used for survival analysis and, Log rank test and Cox proportional hazard test were used for uni and multivariate analysis. Results: From January 2010 to October 2014, there were 69 patients treated with laryngeal preservation and 53 patients received radiation dose 61-70 Gray. After completing radiation, we found that 44 patients have no residual tumor within 6 months and 33 patients can preserve their functional larynx later with complete response (median follow up 6 mo, range 0-46.3 mo). The 1-year, 2-year and 3-year laryngeal preservation rate was 49\%, 36 \% and $32 \%$ respectively. On univariate analysis, lower nodal stage $(\mathrm{p}=0.008)$, stage III disease $(\mathrm{p}=0.046)$, tumor volume $<10 \mathrm{ml}(\mathrm{p}=0.005)$, no true vocal cord involvement $(\mathrm{p}=0.016)$, dose $61-70$ Gray $(\mathrm{p}<0.001)$ and no interruption of treatment $(\mathrm{p}=0.017)$ have better laryngeal preservation rates. ECOG performance status 2, higher nodal stage, stage IV, presence of true vocal cord involvement, upper airway obstruction before/during radiation and radiation dose below 61-70 Gray had an effect on worse overall survival when evaluated with univariate analysis statistical significance. Conclusion: For factors that affected laryngeal preservation in our study were nodal stage, group stage, tumor volume, true vocal cord involvement, radiation dose and treatment break time more than one week with statistical significance.
\end{abstract}

Keywords: Clinical predictor- Laryngeal preservation- Laryngeal Cancer- Hypopharyngeal Cancer- survival outcomes

Asian Pac J Cancer Prev, 20 (7), 2051-2057

\section{Introduction}

Laryngeal cancer is one of the most common Head and Neck cancers. For stage III-IV of this tumor, it can be treated by chemoradiation followed by salvage surgery or surgery and adjuvant radiotherapy (Lefebvre et al., 2010). Surgical treatment of this stage laryngeal cancer often need total removal of the larynx. As a result, patients underwent permanent tracheostomy and had speech and communication problems (Rizzo et al., 2008). These multimodalities are equivalent in terms of survival. However, patients who are treated with upfront chemoradiation can preserve their larynx which extremely affected their quality of life (Lefebvre et al., 2010; Kogashiwa et al., 2009). In 2003, there was a randomized control trial about concurrent chemoradiation for laryngeal preservation, which was updated at the 2006 American Society of Clinical Oncology annual meeting. The study showed that the 5-year laryngeal preservation rate was $83.6 \%$ in concurrent chemoradiation group, $70.5 \%$ in induction chemotherapy group and $65.7 \%$ in radiation alone. However, the overall survival rate and progression free survival rate were not different. Furthermore, the meta-analysis of chemotherapy in Head and Neck cancer demonstrated that concurrent chemoradiation had significant 5-year survival benefit (8\%) compared with radiation alone. In contrast, adjuvant and induction chemotherapy did not improve survival outcome. Consequently, concurrent chemoradiation is likely to be the most appropriate treatment in stage III-IV laryngeal cancer to preserve the larynx (Rizzo et al., 2008). In spite of these benefits, concurrent chemoradiation had considerably acute and late complications, especially dysphagia and recurrent aspirated pneumonia. Therefore, the need to identify the patients who are suitable for upfront surgery or laryngeal preservation by concurrent chemoradiation is very important. Many recent studies revealed that factors related with treatment failure include 
stage, hemoglobin level, male gender, continued smoking and large tumor volume. Rodriguez et al., (2008) explored retrospectively about clinical predictors of successful laryngeal preservation. They found that increased age and continued smoking affected poorer overall survival outcomes. However, there is no study of clinical predictors related with laryngeal preservation rate treated with concurrent chemoradiation in Thailand.

\section{Materials and Methods}

This retrospective study was performed at Department of Radiation Oncology, Rajavithi Hospital, Thailand, From January 2010 to October 2014. The patients who were diagnosed with Laryngeal and hypopharyngeal cancer clinical stage III-IV and treated with organ preservation were assessed retrospectively. Enrollment in these studies was limited to patients with age of 20-70 years, pathological reports-proven squamous cell carcinoma and had medical history, treatment and follow-up records in Rajavithi Hospital. Exclusion criteria were patients who had prior laryngectomy, prior radiation at Head and Neck area, receiving radiation dose less than 45 Gray, had impaired renal function (GFR $<40$ ), had poor liver function test, and poor performance status (ECOG $>2$ ), had evidence of distant metastasis, who were pregnant or lactating and who had two primary cancers. Neoadjuvant chemotherapy is allowed when followed by concurrent chemoradiation.

All patients had a complete blood count, biochemical profile, chest X-ray and Head-and-neck CT scan before treatment. The patients were treated with conventional radiotherapy and standard fractionation, 2 Gray per day, 5 fractions per week. Dose range was 45-70 Gray. They were immobilized in a thermoplastic mask and treated on $6 \mathrm{MV}$ linear accelerator. Dosimetry and treatment planning was performed with Xio version 4.8 with multileaf collimator $1 \mathrm{~cm}$ for two dimensional radiation and three dimensional radiation. The initial treatment volume including primary tumor, involved nodes and potential-microscopic spreading area were treated with 45-50 Gray at least. Dose for gross diseases were escalated to 66-70 Gray. Weekly or triweekly chemotherapy can be adding during radiation. Most patients (71\%) received weekly platinum-based regimen and seven patients had cisplatin or carboplatin-5 FU given continuous infusion Day 1-4 and Day 21-24. Of the 69 patients who were enrolled in the study, only one underwent induction chemotherapy before radiation (Table 1).

Patients were evaluated at monthly intervals as a first follow up, after that every 3-4 months for the first two years and then every4-6 months for the next three years. Chest X-rays were annually examined. Residual disease defined as tumor still seen in originated tumor site within 6 months after completing the treatment. Recurrent tumor defined as presence of tumor more than 6 months after completing the treatment with tumor disappearance before. Suspected obtained sites of residual tumor or locoregional recurrence were evaluated with routine ENT examination and scope, CT scan and confirmed by histopathology. Disease suspected distant metastasis was examined by CT scan, Chest X-rays and bone scan, but was not routinely confirmed by biopsy. Salvage surgery or re-irradiation were considered in a patient with clinical or radiographic evidence of residual disease or locoregional recurrence. Chemotherapy was given in patient who accepted the treatment in case of distant metastasis or locoregional recurrence who cannot be treated with surgery or re-irradiation. In case of poor performance status or refusal of treatment, we treated the patients with best supportive care.

The statistical analysis was performed by SPSS version 17.0. A p-value of less than 0.05 were considered statistically significant. Overall survival, disease free survival and laryngeal preservation survival rate were calculated using the Kaplan-Meier method. Univariate correlation between these factors and patient age, stage, tumor grade, primary site were analyzed by log rank test and multivariate correlation were examined by Cox proportional hazards analysis.

\section{Results}

Of 218 patients, 149 were excluded from the analysis for following reasons: prior glottis surgery (87 patients), withdrawal (7 patients), impaired renal function (7patients), impaired liver function (6 patients, developed metastasis ( 2 patients), developed two primary cancers ( 3 patients), loss of follow up (15 patients) and not fulfill radiation records ( 22 patients). Of the remaining 69 eligible patients, 25 and 44 were stage III and IV respectively. All the distribution of the primary site was 28 larynx and 41 hypopharynx. Of 69 patients, 67 were male and 2 were female, with a median age of 54 years (range 24-76). Patient characteristics are demonstrated in Table 1. Median follow up time was 6 months (range 0-46.3).

The mean Hemoglobin level was $11.4+/-1.7 \mathrm{gm} / \mathrm{dL}$. Most patients $(46.4 \%$ ) had moderately differentiation pathology. Cord fixation and true vocal cord involvement was seen in 18 and 28 patients respectively. Only 15 patients complained of upper airway obstruction before or

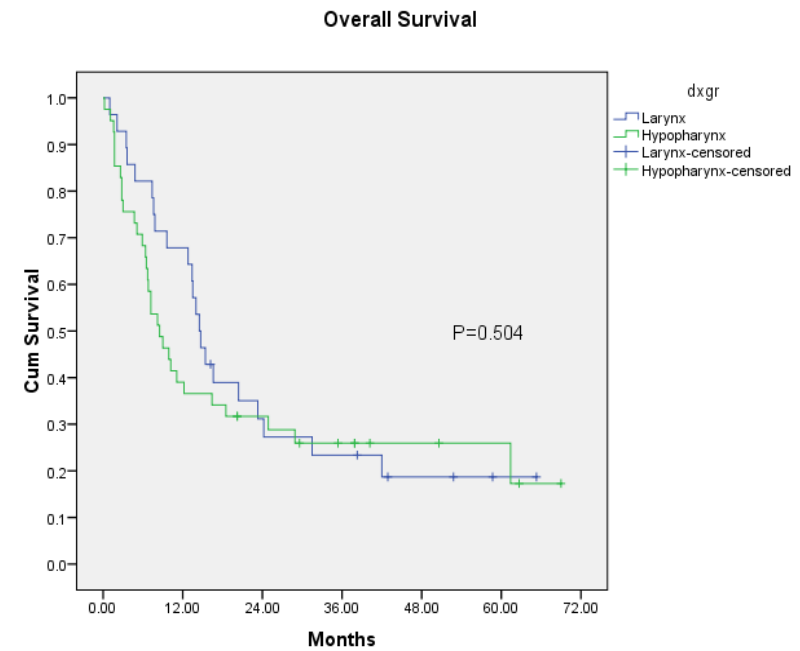

Figure 1. Overall Survival among Stage III-IV Laryngeal and Hypopharyngeal Cancer after treated with organ preservation (subgroup analysis between Laryngeal and Hypopharyngeal cancer. 
Table 1. Baseline Characteristics

\begin{tabular}{|c|c|c|}
\hline Characteristics & & $\mathrm{N}=69$ \\
\hline Age & Median (range) & $54(24-76)$ \\
\hline Gender & $\begin{array}{l}\text { Female } \\
\text { Male }\end{array}$ & $\begin{array}{c}2(2.9 \%) \\
67(97.1 \%)\end{array}$ \\
\hline Co-morbidity & $\begin{array}{l}\text { No } \\
\text { Yes }\end{array}$ & $\begin{array}{l}52(75.4 \%) \\
17(24.6 \%)\end{array}$ \\
\hline $\mathrm{ECOG}^{\mathrm{a}}$ & $\begin{array}{l}0-1 \\
2\end{array}$ & $\begin{array}{l}52(75.4 \%) \\
17(24.6 \%)\end{array}$ \\
\hline Patho & $\begin{array}{l}\mathrm{SCC}^{\mathrm{b}} \\
\text { Non SCC }\end{array}$ & $\begin{array}{c}63(91.3 \%) \\
6(8.7 \%)\end{array}$ \\
\hline \multirow[t]{4}{*}{ Primary site } & Larynx & \\
\hline & $\begin{array}{l}\text { Supraglottic } \\
\text { Glottic } \\
\text { Subglottic }\end{array}$ & $\begin{array}{c}13(18.9 \%) \\
14(20.3 \%) \\
1(1.5 \%)\end{array}$ \\
\hline & Hypopharynx & \\
\hline & $\begin{array}{l}\text { Pyriform sinus } \\
\text { Post cricoid } \\
\text { Posterior pharyngeal wall }\end{array}$ & $\begin{array}{c}28(40.6 \%) \\
8(11.6 \%) \\
5(7.1 \%)\end{array}$ \\
\hline T stage & $\begin{array}{l}\mathrm{T} 1 \\
\mathrm{~T} 2 \\
\mathrm{~T} 3 \\
\mathrm{~T} 4\end{array}$ & $\begin{array}{l}7(10.1 \%) \\
14(20.2 \%) \\
22(31.9 \%) \\
26(37.8 \%)\end{array}$ \\
\hline $\mathrm{N}$ stage & $\begin{array}{l}\text { N0 } \\
\text { N1 } \\
\text { N2 } \\
\text { N3 }\end{array}$ & $\begin{array}{l}22(31.9 \%) \\
18(26.1 \%) \\
18(26.1 \%) \\
11(15.9 \%)\end{array}$ \\
\hline Group stage & $\begin{array}{l}\text { III } \\
\text { IVa-b }\end{array}$ & $\begin{array}{l}25(36.2 \%) \\
44(63.8 \%)\end{array}$ \\
\hline Tumor grade & $\begin{array}{l}\text { Well differentiation } \\
\text { Moderate differentiation } \\
\text { Poorly differentiation } \\
\text { unknown }\end{array}$ & $\begin{array}{c}15(21.7 \%) \\
32(46.4 \%) \\
6(8.7 \%) \\
16(23.2 \%)\end{array}$ \\
\hline True vocal cord involvement & $\begin{array}{l}\text { No } \\
\text { Unilateral } \\
\text { Bilateral }\end{array}$ & $\begin{array}{c}41(59.4 \%) \\
20(29.0 \%) \\
8(11.6 \%)\end{array}$ \\
\hline Cord fixation & $\begin{array}{l}\text { Absence } \\
\text { Presence }\end{array}$ & $\begin{array}{l}51(73.9 \%) \\
18(26.1 \%)\end{array}$ \\
\hline Upper airway obstruction & $\begin{array}{l}\text { Absence } \\
\text { Presence }\end{array}$ & $\begin{array}{c}54(78.3 \%) \\
15(21.7 \%)\end{array}$ \\
\hline Dose & $\begin{array}{l}45-50 \mathrm{~Gy} \\
51-60 \mathrm{~Gy} \\
61-70 \mathrm{~Gy}\end{array}$ & $\begin{array}{c}14(20.3 \%) \\
2(2.9 \%) \\
53(76.8 \%)\end{array}$ \\
\hline Treatment break & $\begin{array}{l}\text { None } \\
\leq 1 \text { wk } \\
>1 \text { wk but }<2 \text { wk }\end{array}$ & $\begin{array}{c}49(71.0 \%) \\
18(26.1 \%) \\
2(2.9 \%)\end{array}$ \\
\hline Technique & $\begin{array}{l}\text { Two-dimensional } \\
\text { Three- dimensional }\end{array}$ & $\begin{array}{c}2(2.9 \%) \\
67(97.1 \%)\end{array}$ \\
\hline $\mathrm{CMT}^{\circ}$ & $\begin{array}{l}\text { Cis/carboplatin weekly } \\
\text { Cis/carboplatin-5FU } \\
\text { Other } \\
\text { None }\end{array}$ & $\begin{array}{c}49(71.0 \%) \\
7(10.1 \%) \\
1(1.4 \%) \\
12(17.5 \%)\end{array}$ \\
\hline Hemoglobin level & Mean +/- SD & $11.4+/-1.7$ \\
\hline Creatinine level & Mean +/- SD & $0.8+/-0.1$ \\
\hline Smoking continuation & $\begin{array}{l}\text { No } \\
\text { Yes }\end{array}$ & $\begin{array}{c}66(95.6 \%) \\
3(4.3 \%)\end{array}$ \\
\hline Alcohol continuation & $\begin{array}{l}\text { No } \\
\text { Yes }\end{array}$ & $\begin{array}{c}68(98.5 \%) \\
1(1.5 \%)\end{array}$ \\
\hline Salvage surgery & $\begin{array}{l}\text { No } \\
\text { Yes }\end{array}$ & $\begin{array}{c}63(91.3 \%) \\
6(8.7 \%)\end{array}$ \\
\hline
\end{tabular}

a, Eastern Cooperative Oncology group(ECOG) performance status scale; b, Squamous cell carcinoma; c, chemotherapy.

during radiation. None of the 69 patients had more than two weeks interruption during radiation. The treatment of 49 patients was not interrupted at all. During radiation,
Disease-Free Survival

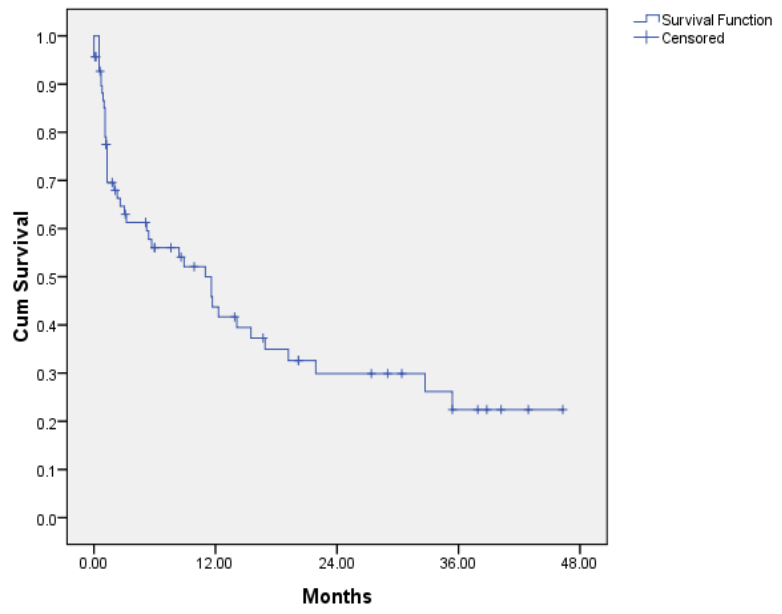

Figure 2. Disease Free Survival among Stage III-IV Laryngeal and Hypopharyngeal Cancer after treated with organ preservation

three of 69 patients continued smoking.

Two dimensional radiation was the treatment for 2 patients $(2.9 \%), 67$ patients $(97.1 \%)$ were treated with three dimensional radiation and none of patients was treated with Intensity Modulated Radiotherapy. Only one patient underwent induction chemotherapy before radiation. For laryngeal cancers, there were 21 patients received weekly regimens, 3 patients received triweekly regimen and 1 patients received other regimen (cetuximab). For hypopharyngeal cancer there were 28 patients received weekly regimens and 4 patients received triweekly regimen. With median follow-up time of 6 months (range $0-46.3$ ), organ preservation controlled the disease with complete response in $14 / 28(50 \%)$ evaluable glottis primaries and 19/41 (46.3\%) evaluable hypopharynx primaries. Of 36 patients with residual disease or recurrent tumor, six were treated with salvage surgery, two were treated with tracheostomy, two were re-irradiated and four were given chemotherapy. In addition 22 patients were treated with best supportive care. In total, 53 of 69 patients received radiation dose of 61-70 Gray. After completing radiation, we found that 44 patients have no residual tumor within 6 months and 33 patients can preserve their functional larynx later with complete response (median follow up time $6 \mathrm{mo}$, range 0-46.3 mo). Of these 69, 36 has residual tumor or developed local relapse and seven were found to have metastasis. Median survival time was 12.2 months. The Kaplan-Meier 1-year, 2-year and 3-year overall survival was $50 \%, 33 \%$ and $25 \%$ respectively (Figure 1). The 1-year, 2-year and 3-year locoregional free survival was $48 \%, 36 \%$ and $31 \%$ respectively. The 1-year, 2-year and 3-year disease free survival was $42 \%$, $30 \%$ and $22 \%$ respectively. The 1-year, 2- year and 3 -year laryngeal preservation rate was $49 \%, 36 \%$ and $32 \%$ respectively. Among the 36 patients with residual disease or local failure, there were 14 larynx primaries and 22 hypopharynx primaries. Local failure developed in 11 patients ( $31 \%$ ) with stage III disease and 25 patients (69\%) with stage IV disease. Permanent tracheostomy 
Table 2. Univariate Analysis of Possible Prognostic Factor

\begin{tabular}{|c|c|c|c|c|}
\hline Variables & & $\mathrm{N}$ & Median survival (mo.) & P-value \\
\hline Age & $\begin{array}{l}>60 \\
<60\end{array}$ & $\begin{array}{l}19 \\
50\end{array}$ & $\begin{array}{l}23.3(6.30,40.26) \\
10.2(5.23,15.16)\end{array}$ & 0.221 \\
\hline $\begin{array}{l}\text { Underlying } \\
\text { disease }\end{array}$ & $\begin{array}{l}\text { No } \\
\text { Yes }\end{array}$ & $\begin{array}{l}52 \\
17\end{array}$ & $\begin{array}{c}9.9(4.48,15.31) \\
14.5(9.66,19.34)\end{array}$ & 0.313 \\
\hline $\mathrm{ECOG}^{\mathrm{a}}$ & $\begin{array}{l}0-1 \\
2\end{array}$ & $\begin{array}{l}52 \\
17\end{array}$ & $\begin{array}{c}14.7(10.98,18.41) \\
7.2(3.16,11.23)\end{array}$ & $<0.001$ \\
\hline $\begin{array}{l}\text { Primary } \\
\text { Site }\end{array}$ & $\begin{array}{l}\text { Larynx } \\
\text { Hypopharynx }\end{array}$ & $\begin{array}{l}28 \\
41\end{array}$ & $\begin{array}{c}14.5(12.0-17.0) \\
8.5(5.3,11.7)\end{array}$ & 0.504 \\
\hline Patho & $\begin{array}{l}\mathrm{SCC}^{\mathrm{b}} \\
\text { Non SCC }\end{array}$ & $\begin{array}{c}63 \\
6\end{array}$ & $\begin{array}{c}12.2(7.86,16.53) \\
6.8(0,45.49)\end{array}$ & 0.709 \\
\hline T stage & $\begin{array}{l}\mathrm{T} 1 \\
\mathrm{~T} 2 \\
\mathrm{~T} 3 \\
\mathrm{~T} 4\end{array}$ & $\begin{array}{c}7 \\
14 \\
22 \\
26\end{array}$ & $\begin{array}{c}\text { Not reach } \\
12.8(6.93,18.66) \\
7.8(0,15.95) \\
7.2(4.70,9.69)\end{array}$ & 0.133 \\
\hline N stage & $\begin{array}{l}\text { N0 } \\
\text { N1 } \\
\text { N2 } \\
\text { N3 }\end{array}$ & $\begin{array}{l}22 \\
18 \\
18 \\
11\end{array}$ & $\begin{array}{c}12.2(4.59,19.80) \\
23.3(11.45,35.15) \\
7.20(5.67,8.72) \\
5.1(0.50,9.69)\end{array}$ & 0.01 \\
\hline Group stage & $\begin{array}{l}\text { III } \\
\text { IV }\end{array}$ & $\begin{array}{l}25 \\
44\end{array}$ & $\begin{array}{c}23.3(11.25,35.34) \\
7.6(4.89,10.30)\end{array}$ & 0.024 \\
\hline Volume & $\begin{array}{l}<10 \mathrm{ml} \\
>=10 \mathrm{ml} \\
\text { unknown }\end{array}$ & $\begin{array}{l}36 \\
21 \\
10\end{array}$ & $\begin{array}{c}16.6(4.67,28.53) \\
8.2(5.21,11.19)\end{array}$ & 0.147 \\
\hline $\begin{array}{l}\text { Cord } \\
\text { Involvement }\end{array}$ & $\begin{array}{l}\text { No } \\
\text { Unilateral } \\
\text { Bilateral }\end{array}$ & $\begin{array}{c}41 \\
26 \\
2\end{array}$ & $\begin{array}{c}16.4(10.17,22.63) \\
7.6(4.60,10.60) \\
3.0\end{array}$ & 0.042 \\
\hline $\begin{array}{l}\text { Cord } \\
\text { Fixation }\end{array}$ & $\begin{array}{l}\text { No } \\
\text { Yes }\end{array}$ & $\begin{array}{l}51 \\
18\end{array}$ & $\begin{array}{c}14.5(10.13,18.87) \\
7.2(5.95,8.45)\end{array}$ & 0.245 \\
\hline $\mathrm{UAO}^{\mathrm{c}}$ & $\begin{array}{l}\text { No } \\
\text { Yes }\end{array}$ & $\begin{array}{l}54 \\
15\end{array}$ & $\begin{array}{c}14.7(10.20,19.20) \\
7.20(6.58,7.82)\end{array}$ & 0.007 \\
\hline Dose & $\begin{array}{l}45-50 \mathrm{~Gy} \\
51-60 \mathrm{~Gy} \\
61-70 \mathrm{~Gy}\end{array}$ & $\begin{array}{c}14 \\
2 \\
53\end{array}$ & $\begin{array}{c}7.2(5.46,8.94) \\
1.6 \\
15.4(9.69,21.11)\end{array}$ & $<0.001$ \\
\hline $\begin{array}{l}\text { Treatment } \\
\text { Break }\end{array}$ & $\begin{array}{l}\text { None } \\
\leq 1 \mathrm{wk} \\
>1-2 \mathrm{wk}\end{array}$ & $\begin{array}{c}49 \\
18 \\
2\end{array}$ & $\begin{array}{c}35.6(9.06,18.94) \\
8.2(3.63,12.77) \\
8.5\end{array}$ & 0.741 \\
\hline Type of CMT ${ }^{\mathrm{d}}$ & $\begin{array}{l}\text { Cis/carbo weekly } \\
\text { Cis/carbo }+5 \mathrm{FU} \\
\text { Other } \\
\text { None }\end{array}$ & $\begin{array}{c}49 \\
7 \\
1 \\
12\end{array}$ & $\begin{array}{c}14.5(10.93,18.07) \\
7.6(0,17.87) \\
14.0\end{array}$ & 0.086 \\
\hline $\begin{array}{l}\text { Smoking } \\
\text { Continuation }\end{array}$ & $\begin{array}{l}\text { No } \\
\text { Yes }\end{array}$ & $\begin{array}{c}66 \\
3\end{array}$ & $\begin{array}{c}12.2(7.82,16.51) \\
7.2\end{array}$ & 0.461 \\
\hline $\begin{array}{l}\text { Alcohol } \\
\text { continuation }\end{array}$ & $\begin{array}{l}\text { No } \\
\text { Yes }\end{array}$ & $\begin{array}{c}68 \\
1\end{array}$ & $\begin{array}{c}11.1(6.55,15.65) \\
16.4\end{array}$ & 0.970 \\
\hline Salvage surgery & $\begin{array}{l}\text { No } \\
\text { Yes }\end{array}$ & $\begin{array}{c}63 \\
6\end{array}$ & $\begin{array}{c}9.9(5.45,14.34) \\
42.0(14.6,69.40)\end{array}$ & 0.073 \\
\hline Recurrence & $\begin{array}{l}\text { No } \\
\text { Yes }\end{array}$ & $\begin{array}{l}33 \\
36\end{array}$ & $\begin{array}{l}14.0(1.55,26.44) \\
10.2(4.76,15.63)\end{array}$ & 0.070 \\
\hline $\begin{array}{l}\text { Larynx } \\
\text { preserve }\end{array}$ & $\begin{array}{l}\text { No } \\
\text { Yes }\end{array}$ & $\begin{array}{l}35 \\
34\end{array}$ & $\begin{array}{l}11.1(5.42,16.77) \\
12.8(1.94,23.65)\end{array}$ & 0.099 \\
\hline $\begin{array}{l}\text { Distant } \\
\text { Metastases }\end{array}$ & $\begin{array}{l}\text { No } \\
\text { Yes }\end{array}$ & $\begin{array}{c}62 \\
7\end{array}$ & $\begin{array}{c}11.1(6.27,15.92) \\
12.8(0,28.45)\end{array}$ & 0.579 \\
\hline
\end{tabular}

a, Eastern Cooperative Oncology group (ECOG) performance status scale; b, Squamous cell carcinoma; c, upper airway obstruction; d, chemotherapy.

was performed in patients or feeding tube dependence was detected in six patients. ECOG performance status 2, higher nodal stage, stage IV, presence of true vocal cord involvement, upper airway obstruction before/during radiation and radiation dose below 61-70 Gray had an effect on worse overall survival when evaluated with 
Table 3. Univariate Analysis of Factor that Affected Laryngeal Preservation

\begin{tabular}{lccc}
\hline Variables & & Median survival mo.) & P-value \\
\hline Group stage & III & $21.9(0.35,43.44)$ & 0.046 \\
& IV & $5.4(1.05,9.74)$ & \\
Volume & $<10 \mathrm{ml}$ & $35.4(5.14,65.65)$ & 0.005 \\
& $>=10 \mathrm{ml}$ & $1.3(0.35,2.25)$ & \\
& unknown & & \\
Cord & No & $15.5(0.90,30.09)$ & 0.016 \\
Involvement & Unilateral & $11.7(6.60,16.79)$ & \\
& Bilateral & 0.5 & \\
N stage & N0 & $12.3(6.50,18.09)$ & 0.008 \\
& $\mathrm{~N} 1$ & $16.9(0-36.25)$ & \\
& $\mathrm{N} 2$ & Not reach & \\
Dose & $\mathrm{N} 3$ & $1.3(0.92,1.68)$ & \\
& $45-50 \mathrm{~Gy}$ & $2.3(0.34,4.26)$ & $<0.001$ \\
& $51-60 \mathrm{~Gy}$ & 0.5 & \\
Treatment & $61-70 \mathrm{~Gy}$ & $19.2(7.63,30.77)$ & \\
Break & $\mathrm{None}$ & $12.3(2.71,21.88)$ & 0.017 \\
& $\leq 1 \mathrm{wk}$ & $11(0,23.16)$ & \\
\hline & $>1-2 \mathrm{wk}$ & 1.1 & \\
\hline
\end{tabular}

univariate analysis statistically significant (Table 2). Whereas there was no statistical significance between age, comorbidity, primary site, T stage, treatment break, type of chemotherapy, smoking history and overall survival rate. We also found that the patients with lower nodal stage $(p=0.008)$, stage III disease $(p=0.046)$, tumor volume $<10 \mathrm{ml}(\mathrm{p}=0.005)$, no true vocal cord involvement $(p=0.016)$, dose 61-70 Gray $(p<0.001)$ and no interruption of treatment $(p=0.017)$ have better laryngeal preservation survival rates on univariate analysis (Table 3). When a multivariate analysis was evaluated ECOG performance status 2 and presence of true vocal cord involvement factor was significantly associated with worse overall survival rate (Table 4). On multivariate

Table 4. Multivariate Analysis of Prognostic Factor for 69 Patients who were Treated with Organ Preservation

\begin{tabular}{|c|c|c|}
\hline & Hazard ratio $(95 \% \mathrm{CI})$ & P-value \\
\hline ECOG $^{\text {a }} 2$ vs $0-1 \mathrm{~N}$ stage & $2.48(1.19-5.17)$ & 0.016 \\
\hline \multicolumn{3}{|l|}{$\mathrm{N}$ stage } \\
\hline No & $\operatorname{Ref}(1)$ & \\
\hline N1 & $0.70(0.22-2.19)$ & 0.545 \\
\hline N2 & $0.49(0.15-1.61)$ & 0.240 \\
\hline N3 & $0.98(0.35-2.71)$ & 0.961 \\
\hline Group stage 4 VS 3 Vocal cord inv. ${ }^{b}$ & $1.33(0.64-2.77)$ & 0.442 \\
\hline \multicolumn{3}{|l|}{ Vocal cord inv. ${ }^{\text {b }}$} \\
\hline None & $\operatorname{Ref}(1)$ & \\
\hline Unilateral & $0.13(0.02-0.70)$ & 0.017 \\
\hline Bilateral & $0.19(0.03-0.94)$ & 0.042 \\
\hline $\mathrm{UAO}^{\mathrm{c}}$ vs none & $1.03(0.44-2.38)$ & 0.936 \\
\hline \multicolumn{3}{|l|}{ Dose } \\
\hline $61-70 \mathrm{~Gy}$ & $\operatorname{Ref}(1)$ & \\
\hline $45-50 \mathrm{~Gy}$ & $1.40(0.52-3.73)$ & 0.496 \\
\hline $51-60 \mathrm{~Gy}$ & $1.53(0.18-12.50)$ & 0.690 \\
\hline
\end{tabular}

a, Eastern Cooperative Oncology group (ECOG) performance status scale; b, vocal cord involvement; c, upper airway obstruction

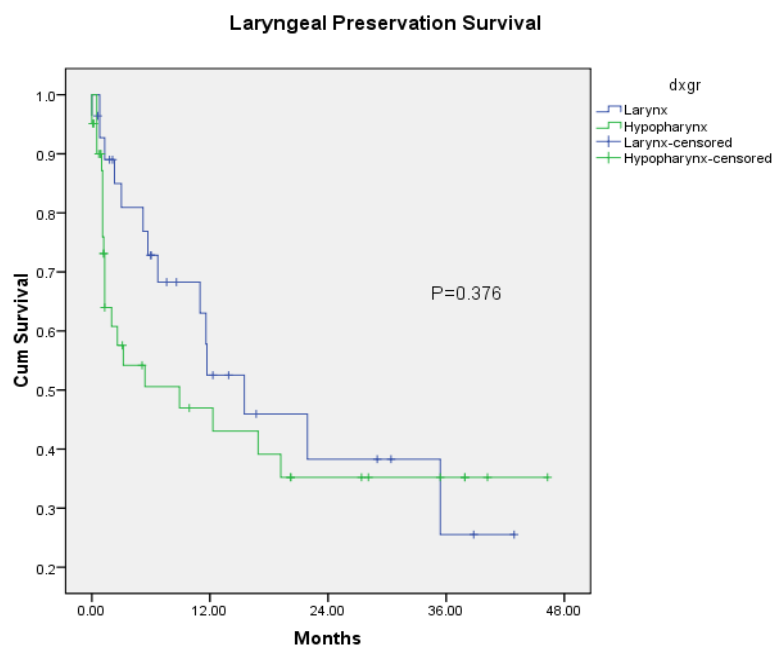

Figure 3. Laryngeal Preservation Rate among Stage IIIIV Laryngeal and Hypopharyngeal Cancer after treated with organ preservation (subgroup analysis between Laryngeal and Hypopharyngeal cancer)

analysis, we also demonstrated that tumor volume more than or equal $10 \mathrm{ml}$ and treatment break more than one week was associated with inferior laryngeal preservation rate statistically significant (Table 5).

\section{Discussion}

The study showed that 1-year, 2- year and 3-year laryngeal preservation rate was $49 \%, 36 \%$ and $32 \%$ respectively. According to our results, laryngeal cancer has no significant difference in overall survival rate when compared with hypopharyngeal cancer (Figure 1).

Kogashiwa et al., (2009) examined laryngeal preservation strategies with induction chemotherapy followed by concurrent chemoradiation in locally advanced laryngeal and hypopharyngeal cancer. They found that laryngeal preservation rate is $93.8 \%$ initially and 3 year rate is $57.8 \%$, which is higher than our study. Also, their findings have better 3 year overall survival rate $(67 \%)$ since their protocol is more intensive than our study (all patients were treated with induction cisplatin and 5FU for two cycles before radiation and followed by concurrent chemoradiation with nedaplatin or docetaxel). Furthermore, their patients had better performance status $(\mathrm{ECOG}<1)$.

Forastiere et al., (2006) investigated about the contribution of adding chemotherapy to radiation in laryngeal preservation strategies. They compared the results of induction cisplatin and 5FU followed by radiation, concurrent chemoradiation with cisplatin and radiation alone. The study showed that 5 year laryngectomy free survival rate is $45 \%$ for sequential chemoradiation, $47 \%$ for concurrent chemoradiation and $34 \%$ for radiation alone $(p=0.011)$. As well as the study of Posner et al., (2009), the 3-year actuarial larynx preservation rate were $70 \%$ with adding of docetaxel to induction chemotherapy regimen and $58 \%$ with PF induction chemotherapy regimen $(p=0.03)$. Whereas, the 3 year laryngeal preservation rate in our study was lower 
Table 5. Multivariate Analysis of Factor that Affected Laryngeal Preservation for 69 Patients who were Treated with Organ Preservation

\begin{tabular}{|c|c|c|}
\hline & Hazard ratio $(95 \% \mathrm{CI})$ & P-value \\
\hline \multicolumn{3}{|l|}{ N stage } \\
\hline N0 & $\operatorname{Ref}(1)$ & \\
\hline N1 & $0.22(0.04-1.27)$ & 0.092 \\
\hline N2 & $0.39(0.07-2.01)$ & 0.261 \\
\hline N3 & $0.19(0.04-0.77)$ & 0.020 \\
\hline Group stage 4 VS 3 & $0.90(0.28-2.81)$ & 0.857 \\
\hline Volume $\geq 10 \mathrm{ml} \mathrm{VS} \mathrm{less}$ & $2.67(1.02-7.01)$ & 0.046 \\
\hline \multicolumn{3}{|l|}{ Vocal cord inv. ${ }^{\mathrm{a}}$} \\
\hline None & $\operatorname{Ref}(1)$ & \\
\hline Unilateral & $0.80(0.04-14.59)$ & 0.881 \\
\hline Bilateral & $2.20(0.13-35.86)$ & 0.577 \\
\hline $\mathrm{UAO}^{\mathrm{b}}$ vs none & $1.03(0.44-2.38)$ & 0.936 \\
\hline \multicolumn{3}{|l|}{ Dose } \\
\hline 61-70 Gy & $\operatorname{Ref}(1)$ & \\
\hline 45-50 Gy & $4.21(0.71-24.85)$ & 0.122 \\
\hline $51-60$ Gy & $<0.001$ & 0.985 \\
\hline \multicolumn{3}{|l|}{ Treatment break } \\
\hline None & $\operatorname{Ref}(1)$ & \\
\hline$\leq 1 \mathrm{wk}$ & $0.07(0.01-0.56)$ & 0.013 \\
\hline$>1-2$ wk & $0.05(0.01-0.40)$ & 0.004 \\
\hline
\end{tabular}

$a$, vocal cord involvement; $b$, upper airway obstruction

(32\%). As there was $17 \%$ of patients who treated with radiation alone in our study. And, all patients was treated with the radiation dose 70 Gray in study of Forastiere et al., (2006) and chemotherapy regimen is triweekly or doublet chemotherapy regimen in the both studies, which is more intensive than ours.

In case of patient who unfit for received chemotherapy during radiation, Cetuximab plus radiation is an alternative choice for organ preservation modalities. Bonner et al., (2005) investigated about laryngeal preservation in locally advanced laryngeal cancer and hypopharyngeal cancer treated with radiation alone compared with Cetuximab plus radiation. They found that the rate of laryngeal preservation was higher when treated with Cetuximab plus radiation. In our study, there are only one patient received Cetuximab during radiation. The other 12 patients who unfit for chemotherapy cannot afford Cetuximab. So the laryngeal preservation rate was lower in our study.

For factors that affected laryngeal preservation in our study were nodal stage, group stage, tumor volume, true vocal cord involvement, radiation dose and treatment break time more than one week with statistical significance. When compared with other studies, the factor that affected on laryngeal preservation rate is also N-stage. Similarly, the study of Ramroth et al., (2011) found that patients with N1-N3 lymph node status was very strong factor on increase in mortality risk. The results showed that N1-N3 status had 3.5 fold increased mortality risk on overall survival. In addition, stage T3 to $\mathrm{T} 4$ have more than twice the mortality rate as much as stage $\mathrm{T} 1$ to $\mathrm{T} 2$. Hence, $\mathrm{T}$ staging is also the prognostic factor that affected survival outcomes. In the same way, Rodrigez et al., (2008) found that patients with early $\mathrm{T}$-stage have better survival outcomes and are treated with salvage surgery less than advanced T-stage with statistical significance. When we calculated the additional factor that impacted on survival outcomes in our study, we also found that patients with T1-T2 stage tended to have more median survival times (23.3 months) than patients with T3-T4 stage (7.8 months), $\mathrm{p}=0.052$.

Furthermore, there are 115 patients with stage II to IV laryngeal and hypopharyngeal cancer in study of Rodrigez et al., (2008). They were treated with conventional fractionated radiation $59 \%$ and hyperfractionated radiation $41 \%$. The total dose was 68-72 Gray and concurrent with cisplatin and $5 \mathrm{FU}$ for two cycles. Hence the treatment is more intensive than our study. They also found that continuation of smoking is one factor that affected laryngeal preservation.

Another factor that affected on laryngeal preservation rate is tumor volume. Strongin et al., (2012) analyzed the relationship between the primary tumor volume and cancer control in squamous cell cancer of the hypopharynx, oropharynx and larynx who treated with definitive chemoradiotherapy. The study demonstrated that tumor volume $<35 \mathrm{~cm}^{3}$ had a better prognosis in terms of progression free survival $(61 \%$ VS $33 \%, \mathrm{p}=0.004)$ and overall survival ( $84 \%$ VS $41 \%, p<0.001)$. Likewise our study, tumor volume (less than $10 \mathrm{ml}$ ) had better laryngeal preservation rate. However, $\mathrm{T}$ stage and $\mathrm{N}$ stage were not significant factor on recurrence or survival outcomes in the study of Strongin et al., (2012), contrast to our study. The possible reason that make this point different when compared to our study is most patients of their study were oropharyngeal cancer (65\%).

The recent study of Grover et al., (2015) investigated the pattern of care and survival outcomes in stage T4a laryngeal cancer treated with total laryngectomy, to compare with laryngeal preservation. The study showed that patients with laryngeal preservation has lower survival rate. On the contrary, the former studies demonstrated that there is no difference in survival aspect between total laryngectomy and laryngeal preservation in locally advanced laryngeal cancer, which may not be applied with stage T4a. Therefore, we implied that stage T4a may affect survival outcomes in laryngeal preservation.

Performance status is mentioned in the study of Grover et al., (2015) at one of the potentially important confounding factors. However, this wasn't recorded in the national cancer database, so the relationship between survival outcomes and performance status was not analyzed.

Moreover, other studies didn't demonstrate the relationship of the radiation dose and survival outcomes because it would increase the confounding effect.

The limitation of our study is the belated follow up of some patients, due to distance from the hospital to their accommodations. Although we plan to have a standard follow up and treatment, some recurrent patients cannot visit as soon as we expected. Their treatment was delayed, so their survival times were less than some patients in other studies. 


\section{Acknowledgements}

The authors wish to thank all the staffs at Department of Radiation Oncology and Department of Otolaryngology - Head and Neck Surgery, Rajavithi Hospital for co-operation, Associate Professor Dr. Srichai Krusun for consultation and Ms.Sam Ormond for her English review.

\section{Funding statement}

This work was supported by a research grant from the Research Committee of Rajavithi Hospital.

\section{References}

Bonner JA, Harari PM, Giralt J, et al (2005). Improved preservation of larynx with the addition of cetuximab to radiation for cancers of the larynx and hypopharynx. J Clin Oncol, 23, 508s.

Enepekides DJ (2005).Concurrent chemoradiotherapy as the most appropriate treatment for most T3 laryngeal carcinomas. Arch Otolaryngol Head Neck Surg, 131, 815-8.

Forastiere AA, Maor M, Weber RS, et al (2006). Long-term results of Intergroup RTOG 91-11: A phase III trial to preserve the larynx-Induction cisplatin/ 5-FU and radiation therapy versus concurrent cisplatin and radiation therapy versus radiation therapy. $J$ Clin Oncol, 24, 284s.

Givens DJ, Karnell LH, Gupta AK, et al (2009). Adverse events associated with concurrent chemoradiation therapy in patients with head and neck cancer. Arch Otolaryngol Head Neck Surg, 135, 1209-7.

Grover S, Swisher-McClure S, Mitra N, et al (2015). Total laryngectomy versus larynx preservation for T4a larynx cancer: Patterns of care and survival outcomes. Int $J$ Radiation Oncology Biol Phys, 92, 594-1.

Kogashiwa Y, Yamauchi K, Nagafuji H, et al (2009). Concurrent chemoradiation for organ function preservation in advanced patients with hypopharyngeal and laryngeal cancer. Oncol Rep, 22, 1163-7.

Lambert L, Fortin B, Soulieres D, et al (2010). Organ preservation with concurrent chemoradiation for advanced laryngeal cancer: Are we succeediong?. Int J Radiation Oncol Biol Phys, 76, 398-2.

Lefebrve JL (2010). Candidates for larynx preservation : The next step ?.Oncologist, 15, 30-2.

Machtay M, Moughan J, Trotti A, et al (2008). Factors associated with severe late toxicity after concurrent chemoradiation for locally advanced head and neck cancer: An RTOG analysis. $J$ Clin Oncol, 26, 3582-9.

Mackenzie RG, Franssen E, Balogh JM, et al (2000). Comparing treatment outcomes of radiotherapy and surgery in locally advanced carcinoma of the larynx : A comparison limited to patients eligible for surgery. Int J Radiation Oncol Biol Phys, 47, 65-1.

Posner MR, Norris CM, Wirth LJ, et al (2009). Sequential therapy for the locally advanced larynx and hypopharynx cancer subgroup in TAX 324: Survival, surgery, and organ preservation. Ann Oncol, 20, 921-7.

Ramroth H, Schoeps A, Rudolph E, et al (2011). Factors predicting survival after diagnosis of laryngeal cancer. Oral Oncol, 47, 1154-8.

Rizzo PB, Maronata F, Marchiori C, et al (2008). Long term quality of life after total Laryngectomy and postoperative radiotherapy versus concurrent chemoradiotherapy for laryngeal preservation. Laryngoscope, 118, 300-6.

Rodriguez CP, Adelstein DJ, Rybicki LA, et al (2008). Clinical predictors of larynx preservation after multiagent concurrent chemotherapy. Head Neck, 30, 1535-2.

Silver CE, Beitler JJ, Shaha AR, et al (2009). Current trends in initial management of Laryngeal cancer: the declining use of open surgery. Eur Arch Otorhinolaryngol, 266, 1333-2.

Strongin A, Yovinno S, Taylor R, et al (2012). Primary tumor volume is an important predictor of clinical outcome among patients with locally advanced squamous cell cancer of head and neck treated with definitive chemoradiotherapy. Int $J$ Radiation Oncol Biol Phys, 82, 1823-9.

Wanebo HJ, Chougoule P, Akerley WL, et al (1997). Preoperative chemoradiation coupled with aggr essive resection as needed ensures near total control in advanced head and neck cancer. Am J Surg, 174, 518-2.

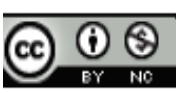

This work is licensed under a Creative Commons AttributionNon Commercial 4.0 International License. 